ISSN electrónico: 2445-1355

DOI: http://dx.doi.org/10.14201/fj202051105106

\title{
ASPECTS OF THE DEVELOPMENT OF NEW ANTI- LEISHMANIA DRUGS: FROM CONTROL OF NEGLECTED INFECTIONS TO PARASITE-HOST INTERACTIONS AND DRUG-RESISTANT PARASITES
}

\section{Sandra GOMES PEREIRA}

CISA-Centro de investigação em Saúde e Ambiente, ESS-IPP, Porto, Portugal

ABSTRACT: Leishmaniasis is a neglected disease caused by parasites of the genus Leishmania. The disease leads to different clinical manifestations determined by parasite features like heterogeneity in the virulence of distinct species-zymodemes and host parameters such as genetic characteristics and immunological status.

Visceral leishmaniasis (VL) is the most severe disease form and is potentially fatal if untreated. Drugs in use against VL present several drawbacks including high toxicity, relevant contraindications and complicated administration regimens. In addition, $90 \%$ of world's VL cases are concentrated in poorest locations like the Indian sub-continent where a number of factors contribute to an ineffective disease control including access to medicines and its relatively expensive price and the development of resistant Leishmania strains, not only to the classical antimonials drugs but also to miltefosine, the only oral drug available.

Therefore, the development of new drugs against VL is urgent and must have in consideration several aspects in order to achieve and be effective to most of the disease cases. Indeed, treatment outcomes vary substantially between different geographic regions and depend on the drug(s) used, drug exposure, severity of disease, host immunity, and the presence of coinfections.

So, in order to improve the drug discovery process for anti-leishmanials the drug screening should involve recent clinical isolates of the L. donovani complex, with different susceptibility profiles to existing drugs and from different geographical origin. 
Both synthetic and natural product libraries might be screened, but evaluation of drugs already in use by developing new formulations or even drug repurposing might also contribute to a faster identification of new candidates. Additional short-term approaches might be combinatory therapies, some of which have proven to be useful mainly in coinfections with HIV.

Other important issue is the establishment of standard techniques for the selection of 'hit compounds' and the criteria established by the Global Health Innovative Technology Fund represent an interesting approach: a given hit should present a 50\% effective concentration (EC50 value) lower than $10 \mu \mathrm{M}$ against intracellular amastigotes of Leishmania sp. and for the in vivo model of VL (i.e. mouse or hamster infected with $L$. infantum or L. donovani), treatment schemes should result in $70 \%$ reduction of liver parasite load after up to 5 doses of $50 \mathrm{mg} / \mathrm{kg}$, orally, one or two times a day.

In the last decades, technology advances have allowed the establishment of High-throughput screening (HTS), a potential efficient way of identifying active compounds able to eliminate the parasite without affecting the host. However, the lack of central database capable of concentrating positive and negative results from different research groups and compounds tested also contributes to delaying the identification of potential candidates against Leishmania protozoa. Also by applying bioinformatic tools such as a systematic in silico chemogenomics strategy in combination with the classical approach, its expected to identify new antiparasitic compounds that will be available for application in the pharmaceutical industry and further research lines.

A relevant portion of drug prospection for neglected diseases relies on academic and research institution but dealing with diseases of such relevance worldwide must require the incorporation of Government and private funding for basic and clinical research projects through direct investments and incentives to both academia and the private sector.

Key words: Parasite; Leishmaniasis; Therapeutics. 